\title{
Prediction of preterm birth using PAMG-1 test: a single centre experience - preliminary report
}

\author{
Wojciech Cnota®, Agnieszka Jagielska®, Ewa Janowska®, Ewa Banas®, \\ Rafal Kierach(1), Malgorzata Nycz-Reska, Bartosz Czuba® \\ Clinical Department of Perinatology, Gynaecology and Obstetrics in Ruda Slaska, \\ Medical University of Silesia, Ruda Slaska, Poland
}

\begin{abstract}
Objectives: Placental alpha microglobulin-1 (PAMG-1) is a novel biomarker detected in cervicovaginal discharge in patients threatened with preterm birth (PTB). This study aimed to show a single centre experience of assessment of imminent spontaneous PTB risk in patients with symptoms suggesting preterm labour (PTL).

Material and methods: The study group consisted of 46 women with singleton pregnancies between $24+0 / 7$ and $33+6 / 7$ weeks of gestation who presented with symptoms of threatened PTL, with cervical dilatation of $<3 \mathrm{~cm}$, cervical length $(\mathrm{CL})$ of $<30 \mathrm{~mm}$ and clinically intact fetal membranes. $\mathrm{CL}$ was measured via transvaginal ultrasound and the PAMG-1 test was performed in all of the objectives.

Results: Sensitivity (SN), specificity (SP), positive predictive value (PPV) and negative predictive value (NPV) of prediction of PTB within seven days for $\mathrm{CL}$ were $100 \%, 11.11 \%, 5.88 \%$ and $100 \%$, respectively. The PAMG-1 test SN, SP, PPV and NPV of the same endpoint were $50 \%, 80.56 \%, 12.5 \%$ and $96.67 \%$, respectively.

Conclusions: PAMG-1 is a more accurate predictor of PTB when compared to CL. Routine use of both mentioned tests could allow identification of low-risk patients and reduction of rate of unnecessary hospitalizations and treatments.

Key words: preterm birth, preterm labou; placental alpha microglobulin-1 (PAMG-1); PartoSure; cervical length (CL)

Ginekologia Polska 2022; 93, 7: 574-577
\end{abstract}

\section{INTRODUCTION}

Preterm birth (PTB) is a delivery between $22+0 / 7$ and $36+6 / 7$ weeks of gestation and is one of the most frequent complications occurring during pregnancy. Preterm labour (PTL) is manifested via regular uterine contractions leading to the cervical shortening or effacement. It affects approximately $5-18 \%$ of pregnancies worldwide [1] and is a leading reason of neonatal mortality and morbidity [2, 3]. Predisposing factors of PTB are: multiple pregnancy, uterus anomaly, previous PTB, cervical insufficiency, ethnicity, low socio-economic status, maternal weight, smoking, periodontal status [4]. Accurate prediction of PTB remains a challenge, whereas more than $2 / 3$ of symptomatic patients do not go on to deliver within seven days from the onset of symptoms [5, 6]. It leads to partly unnecessary admissions to hospital and administrations of tocolytics and antenatal corticosteroids [7], which are not devoid of its adverse consequences [8, 9].
The most widely used but also not the most accurate tool to assess likelihood of PTB is cervical length (CL) measurement via transvaginal ultrasound examination. There are several novel biomarker tests commercially available, based on fetal fibronectin (fFN), phosphorylated insulin-like growth factor-binding protein-1 (phIGFBP-1) and placental alpha microglobulin-1 (PAMG-1) concentrations in cervicovaginal discharge. The most promising test out of the mentioned three seems to be PAMG-1, with reported relatively highest values of sensitivity (SN), specificity (SP), positive predictive value (PPV) and negative predictive value (NPV) [10-15].

\section{Objectives}

This study aimed to show a single centre experience of the Clinical Department of Perinatology, Gynaecology and Obstetrics in Ruda Śląska, Poland of using PAMG-1

\section{Corresponding author:}

Ewa Janowska

Clinical Department of Perinatology, Gynaecology and Obstetrics in Ruda Śląska, Medical University of Silesia, Ruda Śląska, Poland, 2 Lipa St, 41-703 Ruda Śląska, Poland e-mail: e.janowska@outlook.com 
(the PartoSure Test) in assessment of imminent spontaneous PTB risk in patients with symptoms suggesting preterm labour.

\section{MATERIAL AND METHODS}

The prospective study was performed in the period between June 2018 and July 2019 at the Clinical Department of Perinatology, Gynaecology and Obstetrics in Ruda Śląska, Medical University of Silesia, Poland. Forty-six patients, who presented with uterine contractions, were recruited into the study. Inclusion criteria were age of 18 years and above, gestational age between $24+0 / 7$ and $33+6 / 7$ weeks, $C L$ of $<30 \mathrm{~mm}$ in transvaginal ultrasound examination, cervical dilatation of $<3 \mathrm{~cm}$, clinically intact fetal membranes and absence of vaginal bleeding at presentation. As exclusion criteria were used: previous tocolytic therapy, intercourse within the past 24 hours before examination, cervical cerclage or pessary, placenta praevia, multiple pregnancy and patient's disapproval of participation in the study.

A patient who presented to the Department with symptoms of threatened PTL was firstly screened for exclusion criteria, asked for informed consent and then recruited to the study group. A sample for the PAMG-1 test was collected accordingly to the manufacturer's instructions. Then, clinical examination and transvaginal ultrasound with $\mathrm{CL}$ measurement were performed as well as the patient's medical history was collected. The objectives of this study were admitted for a minimum 7-day clinical observation and possible inpatient treatment. A test-to-spontaneous-delivery interval was calculated for all patients.

\section{RESULTS}

There were 46 patients included into the study group initially. Seven were excluded from the final analysis due to lack of follow-up data on the delivery and one due to equivocal PAMG-1 test result, thus 38 objectives represented the study group. The mean age was $30.08 \pm 5.37$ years, the mean gestational age at presentation was $28.61 \pm 2.73$ weeks. A total of $15.79 \%$ (4) of the patients had a history of PTB, $28.95 \%$ (10) of miscarriage (before $22+0 / 7$ weeks of gestation), $34.21 \%$ (13) were taking vaginal progesterone treatment.

\begin{tabular}{|c|c|c|c|c|}
\hline \multirow[b]{2}{*}{$\mathrm{CL}<25 \mathrm{~mm}$} & \multicolumn{2}{|c|}{ Delivery $\leq 7$ days } & \multicolumn{2}{|c|}{ Delivery $\leq 14$ days } \\
\hline & + & - & + & - \\
\hline+ & $2(5.26 \%)$ & $32(84.21 \%)$ & $3(7.89 \%)$ & 31 (81.58\%) \\
\hline - & 0 & 4 (10.53\%) & 0 & 4 (10.53\%) \\
\hline
\end{tabular}

\begin{tabular}{|c|c|c|c|c|}
\hline \multirow[b]{2}{*}{ PAMG-1 } & \multicolumn{2}{|c|}{ Delivery $\leq 7$ days } & \multicolumn{2}{|c|}{ Delivery $\leq 14$ days } \\
\hline & + & - & + & - \\
\hline+ & $1(2.63 \%)$ & $7(18.42 \%)$ & $1(2.63 \%)$ & $7(18.42 \%)$ \\
\hline - & $1(2.63 \%)$ & $29(76.32 \%)$ & $2(5.26 \%)$ & $28(73.68 \%)$ \\
\hline
\end{tabular}

In total $5.26 \%$ (2) of the patients delivered within seven days of presentation and $7.89 \%$ (3) within 14 days. The mean value of $\mathrm{CL}$ was $16.39 \pm 6.44 \mathrm{~mm}$. $92.11 \%$ (34) of the objectives were evaluated as those with a short cervix $(\mathrm{CL}<25 \mathrm{~mm})$, while only two delivered within 7 days and three delivered within 14 days of presentation (Tab. 1). In total, $21.05 \%$ (8) of the study group had a positive result of the PAMG-1 test, out of which one delivered within 7 days of presentation (Tab. 2).

Prediction of delivery within 7 days of testing using CL measurement had sensitivity (SN) of $100 \%$, specificity (SP) of $11.11 \%$, positive predictive value (PPV) of $5.88 \%$ and negative predictive value (NPV) of $100 \%$. SN, SP, PPV and NPV of CL measurement for prediction of delivery within 14 days of testing were $100 \%, 11.43 \%, 8.82 \%$ and $100 \%$, respectively. The PAMG-1 test had SN of $50 \%$, SP of $80.56 \%$, PPV of $12.5 \%$ and NPV of $96.67 \%$ of prediction of delivery within 7 days of testing. Its SN, SP, PPV and NPV of prediction of delivery within 14 days of testing were $33.33 \%, 80 \%$, $12.5 \%$ and $93.33 \%$, respectively (Tab. 3 ).

A test-to-spontaneous-delivery interval ranged from 0 to 109 days, with a mean value of $58.79 \pm 28.32$ days.

\begin{tabular}{|c|c|c|c|c|}
\hline & Sensitivity & Specificity & Positive predictive value & Negative predictive value \\
\hline \multicolumn{5}{|l|}{ Delivery $\leq 7$ days } \\
\hline $\mathrm{CL}<25 \mathrm{~mm}(95 \% \mathrm{Cl})$ & $100 \%(15.81-100 \%)$ & $11.11 \%(3.11-26.06 \%)$ & $5.88 \%(5.28-6.56 \%)$ & $100 \%$ \\
\hline PAMG-1 $(95 \% \mathrm{Cl})$ & $50 \%(1.26-98.74 \%)$ & $80.56 \%(63.98-91.81 \%)$ & $12.5 \%(2.98-39.92 \%)$ & 96.67\% (87.78-99.15\%) \\
\hline \multicolumn{5}{|l|}{ Delivery $\leq 14$ days } \\
\hline $\mathrm{CL}<25 \mathbf{~ m m}(95 \% \mathrm{Cl})$ & $100 \%(29.24-100 \%)$ & $11.43 \%$ (3.2-26.74\%) & 8.82\% (7.91-9.83\%) & $100 \%$ \\
\hline PAMG-1 $(95 \% \mathrm{Cl})$ & $33.33 \%(0.84-90.57 \%)$ & $80 \%$ (63.06-91.56\%) & $12.5 \%(2,47-44.68 \%)$ & 93.33\% (86.08-96.94\%) \\
\hline
\end{tabular}




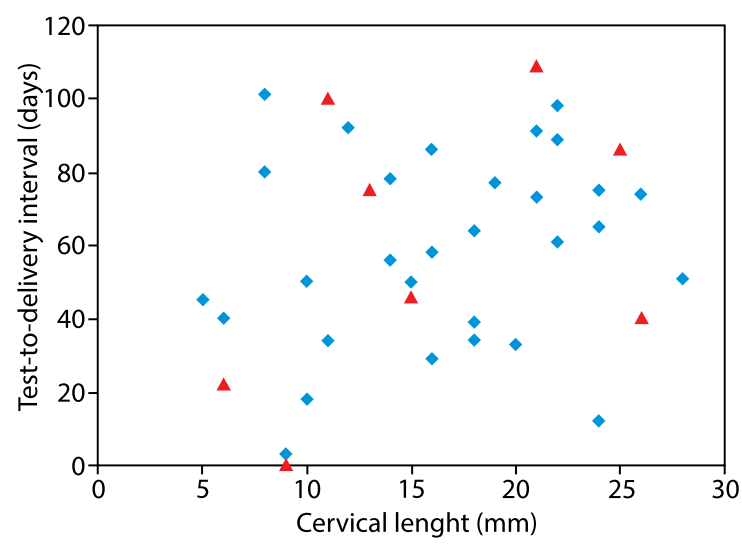

Figure 1. Cervical length $(\mathrm{mm})$ and test-to-spontaneous-delivery interval in the study group. $\mathbf{\Delta}-$ positive result of PAMG-1 test; $\checkmark-$ negative result of PAMG-1 test

Additionally, there was found no statistically significant correlation between $\mathrm{CL}$ and the test-to-spontaneous-delivery interval ( $p=0,1253)$ (Fig. 1).

\section{DISCUSSION}

In the study group only $5.26 \%$ of patients presenting with symptoms suggesting PTL went on to deliver within 7 days. According to the literature, rate of PTB within 7 days of presentation in symptomatic patients ranges from 1.8 to $29.7 \%[5,6]$. It shows that screening for patients truly endangered with PTB is still very poor and leads to partially unnecessary admissions to hospital and treatments [16]. Empana et al. [7], revealed that $85 \%$ of European centres administer multiple courses of antenatal corticosteroids. Although it is proven to be a treatment that promotes neonatal lung maturation, uncontrolled use of corticosteroids may lead to several neonatal adverse consequences, such as respiratory distress syndrome [8] or delay in myelination in the central nervous system [9].

Assessment of likelihood of PTB within 7 days with CL measurement via transvaginal ultrasound in the study group is characterized by high SN and NPV but also very low SP and PPV. Performance of CL measurement in the literature is also unsatisfactory, with SN, SP, PPV, and NPV values of 57\%, $73 \%, 89 \%$ and $30 \%$, respectively [11] and proves $C L$ to be an inaccurate tool in prediction of imminent PTB.

As expected, the PAMG-1 test in the study group obtained a much higher value of SP and similar value of NPV of PTB prediction within 7 days when compared to CL. SN and PPV values of the PAMG-1 test in the study group were not consistent with the literature [17], most likely due to a small size of the group. Nevertheless, obtained data show that PAMG-1 is a superior predictor of imminent PTB than CL.
Since the prediction of PTB in symptomatic patients is still a challenge for clinicians, it needs to provide focus on more efficient screening for patients truly threatened with PTL. Combination of routine CL measurement and the PAMG-1 test could allow identification of patients at low risk of imminent PTL and reduction of rate of unnecessary hospitalizations and antenatal corticosteroids administrations. It would be beneficial not only for patients but also for the healthcare system budget.

\section{CONCLUSIONS}

Our centre experience of predicting imminent spontaneous PTB using the PAMG-1 test is consistent with currently available literature. It shows that the PAMG-1 test is more accurate in assessment of likelihood of PTB than CL measurement. Therefore, routine use of this test should be recommended in order to identify low-risk patients.

\section{Conflict of interest}

All authors declare no conflict of interest.

\section{REFERENCES}

1. Blencowe $\mathrm{H}$, Cousens $\mathrm{S}$, Oestergaard $\mathrm{MZ}$, et al. National, regional, and worldwide estimates of preterm birth rates in the year 2010 with time trends since 1990 for selected countries: a systematic analysis and implications. Lancet. 2012; 379(9832): 2162-2172, doi: 10.1016/S01406736(12)60820-4, indexed in Pubmed: 22682464.

2. Althabe F, Howson CP, Kinney M. World Health Organization Born too soon: the global action report on preterm birth. 2012

3. WARD R. Neonatal complications following preterm birth. BJOG: An International Journal of Obstetrics and Gynaecology. 2003; 110: 8-16, doi: 10.1016/s1470-0328(03)00012-0.

4. Koullali B, Oudijk MA, Nijman TAJ, et al. Risk assessment and management to prevent preterm birth. Semin Fetal Neonatal Med. 2016; 21 (2): 80-88, doi: 10.1016/j.siny.2016.01.005, indexed in Pubmed: 26906339.

5. Sanchez-Ramos L, Delke I, Zamora J, et al. Fetal fibronectin as a short-term predictor of preterm birth in symptomatic patients. Obstetric Anesthesia Digest. 2010; 30(4): 214-215, doi: 10.1097/01.aoa.0000389586.32191.71.

6. Sotiriadis A, Papatheodorou S, Kavvadias A, et al. Transvaginal cervical length measurement for prediction of preterm birth in women with threatened preterm labor: a meta-analysis. Ultrasound Obstet Gynecol. 2010; 35(1): 54-64, doi: 10.1002/uog.7457, indexed in Pubmed: 20014326.

7. Empana JP, Anceschi MM, Szabo I, et al. EURAIL Study Group. Antenatal corticosteroids policies in 14 European countries: factors associated with multiple courses. The EURAIL survey. Acta Paediatr. 2004; 93(10): 1318-1322, indexed in Pubmed: 15499951.

8. Peltoniemi OM, Kari MA, Tammela O, et al. Repeat Antenatal Betamethasone Study Group. Randomized trial of a single repeat dose of prenatal betamethasone treatment in imminent preterm birth. Pediatrics. 2007; 119(2): 290-298, doi: 10.1542/peds.2006-1549, indexed in Pubmed: 17272618.

9. Dunlop S, Archer M, Quinlivan J, et al. Repeated prenatal corticosteroids delay myelination in the ovine central nervous system. Journal of Maternal-Fetal and Neonatal Medicine. 1997; 6(6): 309-313, doi: 10.3109/14767059709162011.

10. Nikolova T, Bayev O, Nikolova N, et al. Evaluation of a novel placental alpha microglobulin-1 (PAMG-1) test to predict spontaneous preterm delivery. J Perinat Med. 2014; 42(4): 473-477, doi: 10.1515/jpm-20130234, indexed in Pubmed: 24334429.

11. Nikolova T, Bayev O, Nikolova $\mathrm{N}$, et al. Comparison of a novel test for placental alpha microglobulin-1 with fetal fibronectin and cervical length measurement for the prediction of imminent spontaneous preterm delivery in patients with threatened preterm labor. J Perinat 
Med. 2015; 43(4): 395-402, doi: 10.1515/jpm-2014-0300, indexed in Pubmed: 25562603.

12. Lee SMi, Romero R, Park JW, et al. The clinical significance of a positive Amnisure test in women with preterm labor and intact membranes. J Matern Fetal Neonatal Med. 2012; 25(9): 1690-1698, doi: 10.3109/14767058.2012.657279, indexed in Pubmed: 22280400.

13. Tripathi R, Tyagi S, Mala YM, et al. Comparison of rapid bedside tests for phosphorylated insulin-like growth factor-binding protein 1 and fetal fibronectin to predict preterm birth. Int J Gynaecol Obstet. 2016; 135(1): 47-50, doi: 10.1016/j.ijgo.2016.03.030, indexed in Pubmed: 27388033.

14. Cooper S, Lange I, Wood S, et al. Diagnostic accuracy of rapid phIGFBP-I assay for predicting preterm labor in symptomatic patients. J Perinatol. 2012; 32(6): 460-465, doi: 10.1038/jp.2011.133, indexed in Pubmed: 21997470.
15. Bolotskikh V, Borisova V. Combined value of placental alpha microglobulin-1 detection and cervical length via transvaginal ultrasound in the diagnosis of preterm labor in symptomatic patients. J Obstet Gynaecol Res. 2017; 43(8): 1263-1269, doi: 10.1111/jog.13366, indexed in Pubmed: 28613021.

16. Lucovnik M, Chambliss LR, Garfield RE. Costs of unnecessary admissions and treatments for "threatened preterm labor". Am J Obstet Gynecol. 2013; 209(3): 217.e1-217.e3, doi: 10.1016/j.ajog.2013.06.046, indexed in Pubmed: 23816842

17. Melchor JC, Khalil A, Wing D, et al. Prediction of preterm delivery in symptomatic women using PAMG-1, fetal fibronectin and phIGFBP-1 tests: systematic review and meta-analysis. Ultrasound Obstet Gynecol. 2018; 52(4): 442-451, doi: 10.1002/uog.19119, indexed in Pubmed: 29920825. 